\title{
PROPER KNOTS IN OPEN 3-MANIFOLDS HAVE LOCALLY UNKNOTTED REPRESENTATIVES
}

\author{
OLLIE NANYES
}

(Communicated by Frederick R. Cohen)

\begin{abstract}
Churchard and Spring [1] conjectured that all proper knots in open 3-manifolds are equivalent to (properly isotopic to) a locally unknotted proper knot. This paper proves the conjecture.
\end{abstract}

\section{INTRODUCTION}

The purpose of this paper is to prove the conjecture made in $\S 3$ of Churchard and Spring [1]; namely, that any proper knot in an arbitrary open 3-manifold is equivalent to (properly isotopic to) a locally unknotted proper knot. However, the suggested construction in [1] does not always work as the following example shows.

Consider the proper knot $K$ defined as ${ }^{*} \times(-1,1)$ in $\mathbf{S}^{2} \times(-1,1)$. Note that this proper knot is not locally unknotted for the following reason: by the "lamp cord" trick, $K$ is ambiently isotopic to a fiber with a trefoil knot tied in it, which is not locally unknotted.

In order to avoid such "global" knotting, we alter a proper knot $K$ within a tubular neighborhood of $K$ into the Fox proper knot [2] via a smooth proper isotopy (Figure 1, p. 564). We then show that this new proper knot, denoted by $K_{f}$, is locally unknotted.

\section{Definitions AND Main theorem}

Unless otherwise stated, all maps and manifolds are assumed to be piecewise linear (or smooth) and all intersections are assumed to be in general position. Let $\mathbf{M}^{3}$ denote an arbitrary open 3-manifold.

Definitions. A map $f: \mathbf{X} \rightarrow \mathbf{M}^{3}$ is said to be proper if for all compact subsets $C, \mathbf{M}^{3} \supseteq C, f^{-1}(C)$ is compact in $\mathbf{X}$. A proper knot $f$ is a proper embedding of $\mathbf{R}^{1}$ into $\mathbf{M}^{3}$. Two proper knots $f_{0}$ and $f_{1}$ are said to be equivalent if there is a proper isotopy $F: \mathbf{R}^{1} \times[0,1] \rightarrow \mathbf{M}^{3}$ such that $F_{0}=f_{0}$ and $F_{1}=f_{1}$. Note that this isotopy need not be an ambient isotopy, but it is either smooth or

Received by the editors February 1, 1990 and, in revised form, April 18, 1990.

1980 Mathematics Subject Classification (1985 Revision). Primary 57M30. 

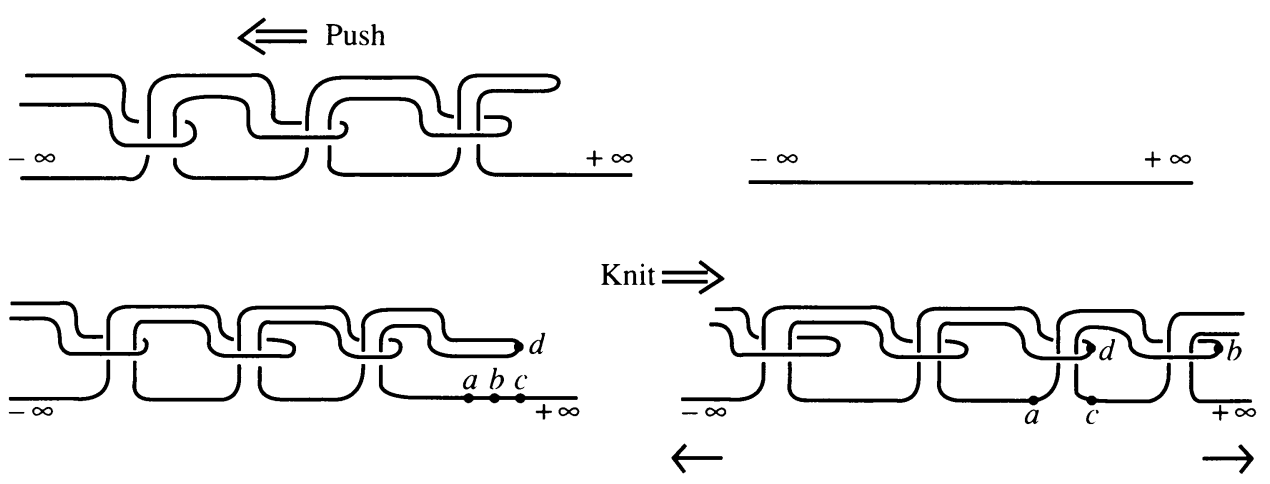

FIGURE 1

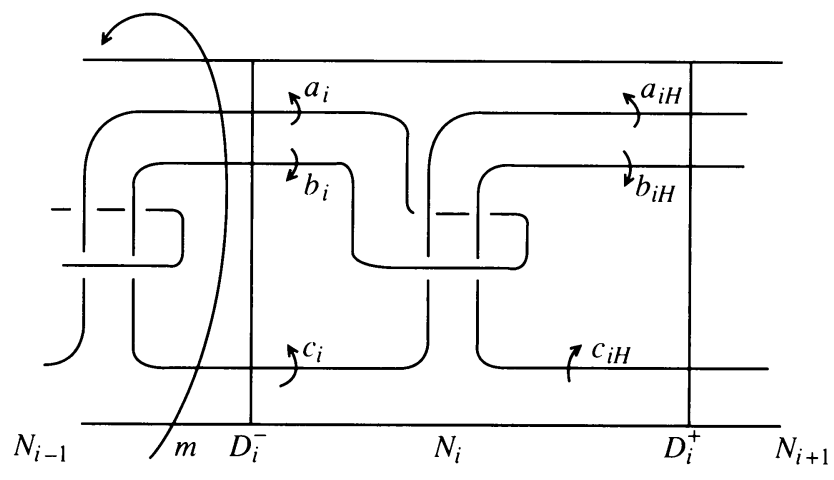

FIGURE 2

locally flat in the p.l. category (that is, the associated level preserving embedding $F \times$ Id: $\mathbf{R}^{1} \times[0,1] \rightarrow \mathbf{M}^{3} \times[0,1]$ is locally flat). By abuse of termiology, I will refer to the image $f\left(\mathbf{R}^{1}\right)$ of a proper knot $f$, denoted by $K$, as a proper knot. A proper knot $K$ is locally unknotted if for every embedded 3-ball $B^{3}$ with $\partial B^{3}$ intersecting $K$ in general position in exactly two points, $B^{3} \cap K$ is unknotted in $B^{3}$. Note: this is equivalent to the existence of a disk $D$ with $\partial D=L \cup P$ (where $P$ is a simple arc on $\partial B^{3}$, and $L=B^{3} \cap K$ ) and $\operatorname{int}(D) \cap B^{3}=\varnothing$. If such a disk $D$ exists for an arc $L$ which intersects a 2 -sphere $S$ in exactly two points, we say that $L$ compresses into $S$. Call a proper knot $K$ strongly locally unknotted if such a $D$ exists for all 2-spheres $S$ (not just those 2-spheres that bound 3-balls) that intersect $K$ in general position in exactly two points. Hence $K$ is locally unknotted if it is strongly locally unknotted.

Let $K$ be an arbitrary proper knot in $\mathbf{M}^{3}$. Let $N$ be a tubular neighborhood of $K$ in $\mathbf{M}^{3}$. In [1] it was shown that $K$ is equivalent to the Fox proper knot $K_{f}$ in $N$ (Figure 2).

Theorem. For any proper knot $K$ in an open 3-manifold $\mathbf{M}^{3}, K_{f}$ is strongly locally unknotted.

One regards $N$ as a union of $D^{2} \times[0,1]$ "chunks" $N_{i},-\infty<i<+\infty$ (Figure 1). In $\partial N_{i}$ let $A_{i}$ denote the annulus $N_{i} \cap \partial N$ and let $D_{i}^{+}, D_{i}^{-}$denote 
the end disks. The intersections of $K_{f}$ with the end disks $D_{i}^{+}$are labeled $a_{i+1}, b_{i+1}$, and $c_{i+1}$ respectively. Note that $D_{i-1}^{+}$is identified with $D_{i}^{-}$. Let $m$ denote the meridian of $N$, i.e., $[m]$ generates $\pi_{1}(\partial N)$. Let $i: \partial N \rightarrow$ $\left(\mathbf{M}^{3}-\operatorname{int}(N)\right)$ be the standard inclusion and $i *: \pi_{1}(\partial N) \rightarrow \pi_{1}\left(\mathbf{M}^{3}-\operatorname{int}(N)\right)$ the induced map on fundamental groups. Note that by the Seifert-Van Kampen theorem:

$$
\pi_{1}\left(\mathbf{M}^{3}-K_{f}\right) \cong \pi_{1}\left(\mathbf{M}^{2}-N\right) * \pi_{1}\left(N-K_{f}\right) /[m]=i *[m] .
$$

Let $H$ the normal closure of the relation $[m]=1$. Let $G$ denote the group $\pi_{1}\left(N-K_{f}\right) / H$. Note that $\pi_{1}\left(N-K_{f}\right) / H$ injects into $\pi_{1}\left(\mathbf{M}^{3}-K_{f}\right)$.

Proposition 1. $G$ is nontrivial.

Proof. First, we calculate $\pi_{1}\left(N_{i}-K_{f}\right)$ by the Wirtinger method. We get the following relations:

$$
\begin{aligned}
& a_{i+1} a_{i} a_{i+1}^{-1}=b_{i+1} b_{i} b_{i+1}^{-1} \\
& b_{i}^{-1}=c_{i} b_{i}^{-1} a_{i+1}^{-1}=c_{i+1} b_{i}^{-1} b_{i+1}^{-1} .
\end{aligned}
$$

Thus we eliminate $c_{i}, c_{i+1}$, and $a_{i}$. We get no other relations. Hence we have that $\pi_{1}\left(N_{i}-K_{f}\right)$ is isomorphic to a free group on three generators and is generated by $\left\langle a_{i+1}, b_{i}, b_{i+1}\right\rangle$. Now, by setting $[m]=1$, we get

$$
[m]=1=b_{i}^{-1} b_{i+1} b_{i} b_{i+1}^{-1} a_{i+1}
$$

which eliminates $a_{i+1}$. We now have that $\pi_{1}\left(N_{i}-K_{f}\right) / H$ is isomorphic to a free group on two generators; namely, $\left\langle b_{i+1}, b_{i}\right\rangle$. Note that this can be seen geometrically. Also note that $\pi_{1}\left(D_{i}^{-}-K_{f}\right) / H$ and $\pi_{1}\left(D_{i}^{+}-K_{f}\right) / H$ are free groups on two generators generated by $\left\langle a_{i}, b_{i}\right\rangle$ and $\left\langle b_{i+1}, c_{i+1}\right\rangle$ respectively.

Lemma 2. We have injections on fundamental groups:

$$
\pi_{1}\left(D_{i}^{-}-K_{f}\right) / H \rightarrow \pi_{1}\left(N_{i}-K_{f}\right) / H \text { and } \pi_{1}\left(D_{i}^{+}-K_{f}\right) / H \rightarrow \pi_{1}\left(N_{1}-K_{f}\right) / H
$$

induced by the natural inclusions.

Proof. Suppose these induced homomorphisms have a nontrivial kernel. Since the image (after replacing $a_{i-1}$ by $b_{i+1} b_{i}^{-1} b_{i+1}^{-1}$ from (1)) of $\pi_{1}\left(D_{i}^{-}-K_{f}\right) / H$ is $\left\langle b_{i}, b_{i}^{-1} b_{i+1} b_{1} b_{i+1} b_{i}\right\rangle$ (respectively the image of $\pi_{1}\left(D_{i}^{-}-K_{f}\right) / H$ is $\left.\left\langle b_{i}, b_{i}^{-1} b_{i+1} b_{i}\right\rangle\right)$ is a subgroup of a need only check that it is not infinite cyclic.

Were it infinite cyclic, then the images of the generators of $\pi_{1}\left(D_{i}^{-}-K_{f}\right) / H$ would be equal in $\pi_{1}\left(N_{i}-K_{f}\right) / H$ (respectively the generators of $\pi_{1}\left(D_{i}^{+}-K_{f}\right) / H$ would be equal in $\left.\pi_{1}\left(N_{i}-K_{f}\right) / H\right)$ since they would be conjugate elements in an abelian group. However this would induce relations in the free group $\pi_{1}\left(N_{i}-K_{f}\right) / H$, which is impossible. 
Now, to prove the proposition, we put in the relations that join the $N_{i}$, we get that $G$ is isomorphic to an infinite free product of free groups of rank two, amalgamated over a free group of rank two, i.e.

$$
G \cong \cdots(Z * Z) *_{Z * Z}(Z * Z) *_{Z * Z}(Z * Z) \cdots .
$$

Therefore $G$ is nontrivial. (It can also be shown that there is a nontrivial representation of $G$ onto a subgroup of $A_{5}$.)

Corollary 3. There is no map $g: \mathbf{S}^{2} \rightarrow \mathbf{M}^{3}$ such that $g\left(\mathbf{S}^{2}\right)$ meets $K_{f}$ in general position in exactly one point (i.e., no singular 2-sphere hits $K_{f}$ in exactly one point).

Proof. First note that $\pi_{1}\left(\mathbf{M}^{3}-K_{f}\right) / H$ has a nontrivial quotient; namely, $\pi_{1}\left(N-K_{f}\right) / H$. Were there such a sphere, then for any generator $b_{i}$, one could constuct a singular disk that is bounded by $b_{i}$ by using a tubular neighborhood of $K_{f}$. This would imply that the above quotient is trivial, contradicting Proposition 1.

Corollary 4. If a disk $C$ is embedded in $N$ in general position with respect to $K_{f}$ and $N$ such that $\partial D$ is nontrivial in $\partial N$ then $C$ hit $K_{f}$ in at least three points.

Proof. Because $H_{1}\left(N-K_{f}\right) \cong Z, C$ must meet $K_{f}$ in an odd number of points. However, $C$ cannot hit $K_{f}$ only once, as $\partial C$ corresponds to $[m]$, which is trivial in $G$ whereas $G$ is nontrivial.

We are now ready to start the proof of our theorem. Our strategy is as follows: we let $S$ be any two sphere in our arbitrary open 3-manifold $\mathbf{M}^{3}$ that hits $K_{f}$ in general position in exactly two points, say $x_{0}$ and $x_{1}$. We successively modify $S$ in order to remove as many components of intersection as possible of $S$ with $\partial N$ into a new two sphere $S^{\prime}$ in such a way that if $K_{f}$ had a subarc (with endpoints on $S^{\prime}$ ) which compressed into $S^{\prime}$, then the same subarc compressed into $S$. We then show that $S^{\prime} \cup N$ with its regular neighborhood in $\mathbf{M}^{3}$, denoted by $R\left(S^{\prime} \cup N\right)$, embeds into $\mathbf{S}^{2} \times(-1,1)$ via some p.l. homeomorphism $\Phi$. Then we show that $K_{f}$ is strongly locally unknotted in $\mathbf{S}^{2} \times(-1,1)$. Then, because $\mathbf{S}^{2} \times(-1,1)$ is actually (i.e., can be identified with) the union of $\Phi\left(R\left(S^{\prime} \cup N\right)\right)$ and a finite number of 3-balls (the end two being half open) that are attached to $\Phi\left(R\left(S^{\prime} \cup N\right)\right)$ via a neighborhood (on $\partial N$ ) of meridional "attaching 1-spheres" and boundary disk components of $\Phi\left(R\left(S^{\prime}-N\right)\right)$, the "compressing disk" can be ambiently isotoped into $\Phi\left(R\left(S^{\prime} \cup N\right)\right)$. It then can be mapped homeomorphically via $\Phi^{-1}$ into $\mathbf{M}^{3}$ where it still meets the necessary requirements (i.e. its boundary is still a union of subarcs on $S^{\prime}$ and $K_{f}$ with endpoints $x_{0}$ and $x_{1}$ ). Note: henceforth, all maps and intersections are assumed to be in general position.

Lemma 5. (The disk swapping lemma). Let $K$ be an arc, $S$ a two sphere, $K$ and $S$ p.l. (or smoothly) embedded in an open 3-manifold $M, K \cap S=$ $\left\{x_{0}, x_{1}\right\}$ a two-point set. Let $J$ be any disk in $S$ that misses $x_{0}$ and $x_{1}$. 
Suppose $J$ is replaced by another disk $J^{\prime}, M \supseteq J^{\prime}, J^{\prime} \cap K=\varnothing$, such that $\partial J^{\prime},\left(J^{\prime}-\partial J^{\prime}\right) \cap(S-J)=\varnothing$ to yield a modified sphere $S$. If $K$ compresses into the modified sphere $S^{\prime}$, then it compresses into $S$.

Proof. With no loss of generality we may assume that the compressing disk $\Delta$ is bounded by $K \cup \alpha$ where $\alpha$ is an arc that misses $J^{\prime}$ (we can isotope $\Delta \cap S^{\prime}=\alpha$ to any other arc in $S^{\prime}$ with endpoints $x_{0}$ and $x_{1}$ ). Now assume that $\operatorname{int}(J)$ intersects $\Delta$. If it does not, we are done. The components of intersection are simple closed curves on $\Delta$ and $J$. Choose one that is innermost on $J$, say $\gamma$. $\gamma$ is also a simple closed cure on $\Delta$. We now modify $\Delta$ by slightly enlarging $\gamma$ and then replacing the disk bounded by the enlarged $\gamma$ by a disk missing $J, S^{\prime}$, and $K$. (This can be thought of as a "parallel" copy of the replaced disk.) This reduces the number of components of $J \cap \Delta$ by at least one, and by compactness there must have been only a finite number of components to begin with. Continue this process until all components of intersection are removed. The lemma is proved.

Let $S$ be any two sphere that intersects $K_{f}$ in exactly two points. Consider $S \cap \partial N$. This is a finite collection of simple closed curves on both $S$ and $\partial N$. We call a simple closed curve $\gamma$ trivial if it is homotopically trivial on $\partial N$. We call $\gamma$ separating if it separates $x_{0}$ and $x_{1}$ on $S$.

Proposition 6. $S$ can be modified via disk swaps so that there are no separating simple closed curves in $S \cap \partial N$.

Proof. We will prove this in four steps. Note: only Step 2 involves a modification of $S$; the other steps assert the nonexistence of various types of simple closed curves.

Step 1. There can be no separating, trivial simple closed curves. Suppose one existed, say $\alpha . \alpha$ then bounds a disk on $\partial N$ and a disk on $S$, which we may assume contains $x_{0}$ (since it is a separating curve). These two disks can be joined along $\alpha$ to form a sphere that hits $K_{f}$ in exactly one point, $x_{0}$. This contradicts Corollary 3.

Step 2. Any trivial simple closed curve can be removed by a disk swap. Let $\alpha$ be a trivial simple closed curve that is innermost on $\partial N$. It bounds a disk $F^{\prime}$ on $\partial N$. By Step 1, $\alpha$ must bound a disk on $S$ that does not contain either $x_{0}$ or $x_{1}$. Call this disk $F$. We can then swap an appropriately "pushed out" copy of $F^{\prime}$ for $F$, since neither disk meets $K_{f}$. Note that $\alpha$ need not have been innermost on $S$. Proceeding inductively, one removes the finite number of trivial simple closed curves on $\partial N$. Therefore, all simple closed curves of intersection are meridional on $\partial N$.

Step 3. There can be no innermost (on $S$ ), nontrivial sseparating simple closed curves of intersection.

Suppose $\alpha$ is such a curve. $\alpha$ then bounds a disk $F$ on $S$ that contains exactly one point of $K_{f}$, say $x_{0}$. Note that $F$ must lie entirely in $N$ as $\alpha$ is innermost. Then $F$ is a disk such that $\partial F$ is on $\partial N(\partial F=\alpha)$ that hits $K_{f}$ 
in only one point. This contradicts Corollary 4.

Step 4. There can be no nontrivial, separating simple closed curves of intersection.

Suppose $\alpha$ is such a curve. $\alpha$ then bounds a disk $F_{0}$ on $S$ containing exactly one point of $K_{f}$, say $x_{0} \cdot \alpha$ also bounds a disk $F_{1}$ on $S$ containing $x_{1}$ and no other point of $K_{f}$. By Step 3, $\alpha$ is not innermost on $S$, therefore, there is an innermost simple closed curve of intersection, on say $F_{1}$, say $\beta$, which necessarily (by Step 3 it cannot be separating) bounds a disk $F_{1}^{\prime}$, on $F_{1}$ which contains no point of $K_{f}$. By Step 2, $\beta$ must be meridional on $\partial N$. Hence, $\beta$ and $\alpha$ bound an annulus on $\partial N$, called $A^{\prime}$. Therefore, we may obtain a 2-sphere, possibly singular, by joining $F_{0}$ along $\alpha$ to the annulus (a suitable pushed copy) $A^{\prime}$ which is attached to the disk $F_{1}^{\prime}$, along $\beta$. The existence of this 2-sphere (possibly singular) contradicts Corollary 3 since it meets $K_{f}$ in exactly one point, $x_{0}$. This completes Step 4 and the proof of the proposition.

Proposition 7. $S$ can be modified so that all simple closed curves of intersection are innermost on $S$.

Proof. There are only a finite number of simple closed curves of intersection and all are meridional on $\partial N$. In addition, all must bound disks on $S$ which do not meet $K_{f}$ by the previous proposition. By finiteness, there must be on $\partial N$ an adjacent pair of simple closed curves of intersection, one of which is innermost on $S$ call it $\alpha$, and one that is not innermost on $S$, say $\beta$ (provided there are any noninnermost ones !eft). Call the annulus between $\alpha$ and $\beta$ on $\partial N A^{\prime}$. Now there are disks on $S$ bounded by $\alpha$ (resp. $\beta$ ) $F_{\alpha}$ (resp. $F_{\beta}$ ) which are disjoint from $K_{f}$. Then we may perform a disk swap which replaces $F_{\beta}$ by a slightly pushed out copy of $A^{\prime}$ which is joined to an appropriately modified (pushed out) copy of $F_{\alpha}$. This ensures that $\beta$ becomes an innermost curve of intersection. Note: all the modifications can take place in an appropriately small regular neighborhood of $S \cup N$. Also notice that we did not alter the intersection of $S$ with $K_{f}$ in any way. This proves the proposition.

Proposition 8. $S$ can be modified so that $S \cup N$ and its regular neighborhood in $\mathbf{M}^{3}$ can be embedded into $\mathbf{S}^{2} \times(-1,1)$.

Proof. There are two cases. The first case has $S$ not intersecting $\partial N$ at all. In this case $S$ is contained in $N$ and $N$ can be embedded into $\mathbf{S}^{2} \times(-1,1)$ as a regular neighborhood of a fiber ${ }^{*} \times(-1,1)$ where ${ }^{*} \in \mathbf{S}^{2}$. Now suppose that the intersection of $S$ and $\partial N$ is not empty. From the previous propositions, the intersection consists of a finite collection of simple closed curves, say there are $k$ of them, $\alpha_{i}, 1 \leq i \leq k$, each of which is meridional on $\partial N$. Furthrmore, each of the $\alpha_{i}$ are innermost on $S$ and bound a disk $F_{i}$ on $S$ which does not intersect $K_{f}$. Hence, the interiors of each of the $F_{i}$ lie outside of $N$. Order the $\alpha_{i}$ according to their least $\mathbf{R}^{1}$ coordinate with the first one labeled $\alpha_{1}$. We denote the annulus on $\partial N$ between $\alpha_{i}$ and $\alpha_{i+1}$ by $A_{i}$. Now go to $\alpha_{2}$. If necessary, we can replace $F_{2}$ by a slightly modified $A_{1}$ joined to a slightly 
modified $F_{1}$. We can repeat this process for all $i$ until all of the new disks bounded by the $\alpha_{i}$ are parallel. Now a regular neighborhood of this modified $S \cup N$, denoted by $R(S \cup N)$, is homeomorphic to a copy of $\mathbf{D}^{2} \times \mathbf{R}^{1}$ with a finite number of 2-handles attached along meridional attaching 1-spheres, the $\alpha_{i}$. (The cores of the handles are the $F_{i}$.) Then, letting the $F_{i}$ correspond to the $\mathbf{S}^{2}$ factor, we can use any homeomorphism (p.l. or smooth) between $\mathbf{S}^{2} \times \mathbf{R}^{1}$ embedding of $R(S \cup N)$ into $\mathbf{S}^{2} \times(-1,1)$ by $\boldsymbol{\Phi}$. Note that in $\mathbf{S}^{2} \times(-1,1)$, we can assume that

$$
\Phi(R(S \cup N))=(\mathbf{D} \times(-1,1)) \bigcup_{i=1}^{k}\left(\mathbf{D}_{\mathbf{C}} \times\left(t_{i}-\varepsilon, t_{i}+\varepsilon\right)\right),
$$

(where $\mathbf{D}$ is a disk in the $\mathbf{S}^{2}$ factor which corresponds to $\Phi\left(D_{i}^{+}, \mathbf{D}_{\mathbf{C}}\right.$ is its complementary disk in the $\mathbf{S}^{2}$ factor and $t_{i}$ corresponds to the $(-1,1)$ factor associated with $\Phi\left(\alpha_{i}\right)$, and $\mathbf{D}_{\mathbf{C}} \times t_{i}$ corresponds to the $\Phi\left(F_{i}\right)$.) In addition, $\left(\mathbf{S}^{2} \times(-1,1)\right)-\Phi\left(R(S \cup N)\right.$ is now a union of $k+13$-balls, $h_{i}$, and $h_{1}$ and $h_{k+1}$ being half open. The boundaries of the $h_{i}$ are the following:

$$
\begin{aligned}
& \text { for } i=1, \quad \partial h_{i}=\left(\partial \mathbf{D} \times\left[-1,\left(t_{1}-\varepsilon\right)\right]\right) \cup\left(\mathbf{D}_{\mathbf{C}} \times\left(t_{1}-\varepsilon\right)\right), \\
& \text { for } i=2 \text { to } k, \\
& \quad \partial h_{i}=\left(\partial \mathbf{D} \times\left[\left(t_{i-1}+\varepsilon\right),\left(t_{i}-\varepsilon\right)\right]\right) \cup\left\{\left(\mathbf{D}_{\mathbf{C}} \times\left(t_{i-1}+\varepsilon\right)\right) \cup\left(\mathbf{D}_{\mathbf{C}} \times\left(t_{i}-\varepsilon\right)\right)\right\}, \\
& \text { for } i=k+1, \quad \partial h_{i}=\left(\partial \mathbf{D} \times\left[\left(t_{k}+\varepsilon\right), 1\right]\right) \cup\left(\mathbf{D}_{\mathbf{C}} \times\left(t_{k}+\varepsilon\right)\right) .
\end{aligned}
$$

Henceforth, when working in $\mathbf{S}^{2} \times(-1,1)$, I will suppress " $\Phi$ ” when referring to the image of $S$ and $N$.

Proposition 9. Let $K$ be any proper knot running between the opposite ends of $\mathbf{S}^{2} \times(-1,1)$. Let $S^{\prime}$ be any 2-sphere that either misses $K$ or intersects $K$ in an even number of points. Then $S^{\prime}$ bounds a ball in $\mathbf{S}^{2} \times(-1,1)$.

Proof. $S^{\prime}$ separates in $\mathbf{S}^{2} \times(-1,1)$. If $S^{\prime}$ separated the ends of $\mathbf{S}^{2} \times(-1,1)$ from one another, $S^{\prime}$ would hit $K$ in an odd number of points. Hence $S^{\prime}$ bounds in $\mathbf{S}^{2} \times(-1,1)$ and therefore $s^{\prime}$ bounds a ball.

Proposition 10. $K_{f}$ is strongly locally unknotted in $\mathbf{S}^{2} \times(-1,1)$.

Proof. First note that the boundaries of the end disks of the chunks $N_{i}, \partial D_{i}$ (I will work with $D_{i}^{+}$and suppress the " $+D$ ") bound disks $P_{i}$ in the complement of $N$ in $\mathbf{S}^{2} \times(-1,1)$. We can then form spheres $S_{i}=P_{i} \bigcup_{\partial D_{i}} D_{i}$. We can then form modified chunks $N_{i}^{\prime}$ whre $N_{i}^{\prime}$ is that part of $\mathbf{S}^{2} \times(-1,1)$ that is bounded by the "boundary spheres" $S_{i-1}$ and $S_{i}$. (i.e. $N_{i}^{\prime}$ is obtained by gluing a " $P_{i} \times[0,1]$ " to $N_{i}$ along $\partial N_{i}$ in a standard way). It follows from Lemma 2 that $\pi_{1}\left(S_{i}-K_{f}\right)$ injects into both $\pi_{1}\left(N_{i}^{\prime}-K_{f}\right)$ and $\pi_{1}\left(N_{i+1}^{\prime}-K_{f}\right)$.

We start the proof of our proposition by showing that we can ambiently isotope $S$ into a single $N_{i}^{\prime}$ in such a manner that $K_{f}$ remains setwise fixed. Note that by compactness, $\left(\bigcup_{i}^{\infty} S_{i}\right) \cap S$, is a finite collection of simple closed 
curves on the $S_{i}$ and on $S$. Choose one that is innermost on $S$, say $\beta$. $\beta$ bounds an innermost disk $F$ on $S$ and two disks on a $S_{i}$. We show that all such curves can be removed. This process must terminate after a finite number of steps. We start off with those $F$ that either contain either no or one point of $K_{f}$. Note that after these innermost simple closed curves of intersection are removed, there will be no $F$ that contains two points of $K_{f}$.

Case $1 . F$ does not contain a point of $K_{F}$. Now $\beta$ bounds two disks on $S_{i}$. Neither disk can contain exactly one point of $K_{F}$ because if one disk did, say $C$, then $F \bigcup_{\beta} C$ would form a 2-sphere that hits $K_{f}$ exactly once; the existence of such a sphere contradicts Corollary 3. Thus $\beta$ bounds a disk $C$ on $S_{i}$ which contains no points of $K_{f}$. By Proposition 9, the sphere formed by $F \bigcup_{\beta} C$ bounds a ball which can be used to guide an ambient isotopy which removes $\beta$ and fixes $K_{f}$.

Case 2. $F$ contains one point of $K_{f}$ Consider $\beta$ on $S_{i} . \quad \beta$ cannot bound a disk $C$ on $S_{i}$ which misses $K_{f}$ else $F \bigcup_{\beta} C$ would form a 2-sphere hitting $K_{f}$ in one point, contradicting Corollary 3 . Hence $\beta$ bounds a disk $C$ on $S_{i}$ that contains one point of $K_{f}$. Thus $F \bigcup_{\beta} C$ hits $K_{f}$ twice and thus bounds a ball $B$ in $N_{i}^{\prime}\left(0 \mathrm{r} N_{i+1}^{\prime}\right)$. Employing the following lemma one easily shows that $B$ can be used to guide an ambient isotopy which fixes $K_{F}$ setwise and removes $\beta$.

Lemma 11. Let $B$ be any 3-ball contained in a single $N_{i}^{\prime}$ whose boundary intersect $K_{f}$ twice. Then $B \cap K_{f}$ is unknotted.

Proof. Because $[m]=1$ in $\mathbf{S}^{2} \times(-1,1)$, as well as in $N_{i}^{\prime}$,

$$
\pi_{1}\left(N_{i}^{\prime}-K_{f}\right) \cong \pi_{1}\left(N_{i}-K_{f}\right) / H \cong Z^{*} Z \text {. }
$$

I will denote this group by $W$. $W$ contains no subgroup that is isomorphic to a nontrivial knot group; $W$ is a free group and thus all of its subgroups are free. But the only free knot group is the trivial knot group. By the Seifert-Van Kampen theorem:

$$
W \cong \pi_{1}\left(B-K_{f}\right)^{*} \pi_{1}\left(\left(N_{i}^{\prime}-B\right)-K_{f}\right) / i *[n]=[n],
$$

where $n$ is the meridian of $\left(B \cap K_{f}\right)$ and $i *$ is induced by the inclusion of $\left(\partial B-K_{f}\right)$ into $N_{i}^{\prime}$. Because $i *[n]$ is either $b_{i}$ or $b_{i+1}$, neither which is trivial in $W$, the knot group of $\left(B \cap K_{f}\right)$ is a nontrivial subgroup of $W$. Because it is both a knot group and a free group, it must be the trivial knot group. Lemma 11 is proved.

We have now shown that all innermost (on $S$ ) simple closed curves of intersection of $S$ with the $S_{i}$ can be removed. Hence, we may assume that $S$ lies entirely in one modified chunk $N_{i}^{\prime}$. Becuase $S$ hits $K_{f}$ twice, we can now apply Lemma 11 to show that, if $B$ is the ball in $\mathbf{S}^{2} \times(-1,1)$ bounded by $S$ (which evidently lies in $N_{i}^{\prime}$ ), $B \cap K_{f}$ is unknotted. Since $S$ was only changed by ambient isotopies, we can assume that $K_{f}$ compresses into the original (unmodified) embedded $S$. Proposition 10 is proved. 
We are ready to prove our main theorem. The following proposition will show that a "compressing" disk bounded by a subarc of $K_{f}$ and a subarc of $S$ can be ambiently isotoped into $\Phi(R(S \cup N))$. The disk can then be lifted back to $\mathbf{M}^{3}$ where it meets the necessary requirements. Let $\Delta$ be the disk that $K_{f}$ uses to compress into $S$.

Proposition 12. $\Delta$ can be isotoped into $\Phi(R(S \cup N))$.

Proof. Recall the construction of $\mathbf{S}^{2} \times(-1,1)$ as given at the end of Proposition 8. Consider the intersection of the interior of $\Delta$ with the $\partial h_{i}(\partial \Delta$ is disjoint from the $h_{i}$ ). The components of intersection are simple closed curves on $\partial h_{i}$ and on $\Delta$. Choose one that is innermost on a $\partial h_{i}$, say $\gamma . \gamma$ bounds a disk on $\partial h_{i}$ and on $\Delta$. One then can replace the disk on $\Delta$ by a suitably pushed out copy of the disk on $\partial h_{i} . \Delta$ is still a disk and its boundary has not been affected. This process terminates after a finite number of steps. Thus, $\Delta$ is modified to miss the $h_{i}$ and therefore lies in $\Phi(R(S \cup N))$. The proposition is proved.

\section{ACKNOWLEDGMENTS}

I would like to thank Cameron Gordon and the referee for their advice concerning this paper.

\section{REFERENCES}

1. P. Churchard and D. Spring, Proper knot theory in open 3-manifolds, Trans. Amer. Math. Soc. 308 (1988), 133-142.

2. R. H. Fox, A remarkable simple closed curve, Ann. of Math. 50 (1949), 264-265.

3. R. H. Fox and E. Artin, Some wild cells and spheres in three dimensional space, Ann. of Math. 49 (1948), 979-990.

4. D. Rolfsen, Knots and links, Publish or Perish, 1976.

Department of Mathematics, University of Texas at Austin, Austin, TeXas 78712 